\title{
A PEGAdA ECOLÓGICA EMERGÉTICA COMO INSTRUMENTO DE AVALIAÇÃo DA SUSTENTABILIDADE AMBIENTAL
}

\author{
Wallan Azevedo dos SANTOS ${ }^{1}$, Glaucio José Pereira da SILVA ${ }^{1}$, Henrique Rego Monteiro da HORA H $^{1}$ \\ Milton ERTHAL JR ${ }^{1,2}$. \\ ${ }^{1}$ Instituto Federal de Educação, Ciência e Tecnologia Fluminense. Campos dos Goytacazes, Rio de Janeiro Brasil. \\ 2 Universidade Candido Mendes. Campos dos Goytacazes, Rio de Janeiro, Brasil. \\ *Autor para correspondência: miltonerthal@ hotmail.com
}

DOI: http://dx.doi.org/10.18571/acbm.175

\section{RESUMO}

Este artigo tem o objetivo realizar uma análise bibliométrica sobre o Método da Pegada Ecológica Emergética como instrumento de avaliação da sustentabilidade ambiental. A pesquisa partiu de um levantamento de dados bibliográficos na base Scopus, utilizando-se de técnicas bibliometricas com devidos refinamentos. A análise considerou os periódicos com maior número de publicações, principais autores, nacionalidade e instituições mais relevantes, identificação das palavras-chave mais comuns e artigos mais citados. Foram encontrados 138 documentos, sendo 87 artigos científicos que passaram por uma análise mais aprofundada. A área de Ciências Ambientais foi responsável por $81 \%$ dos artigos publicados. O periódico com mais artigos publicados foi o Ecological Indicators, embora o Journal of Cleaner Production tenha apresentado melhores índices de desempenho bibliométricos. A China e os Estados Unidos da América são os países com o maior número de publicações e, no caso brasileiro, a Universidade Estadual de Campinas é a instituição com o maior número de filiações. Conclui-se que o tema é relativamente recente, visto que as publicações se iniciaram a partir de 2003, com concentração expressiva entre os anos de 2011 e 2015, quando o número de publicações quadruplicou. A temática abordada neste estudo apresentou alto índice de replicabilidade em diferentes esferas de análises de sustentabilidade.

Palavras-chave: Bibliometria; Base Scopus; Gestão Ambiental; Sustentabilidade.

\begin{abstract}
This article aims to perform a bibliometric analysis on the theme "Ecological Footprint Method based on the concepts of emergy". The research was based on a survey of bibliographic data in the Scopus database, using bibliometric techniques with due refinements. The analysis considered the journals with the largest number of publications, main authors, nationality and most relevant institutions, identification of the most common keywords and most cited articles. 138 documents were found, which 87 were scientific articles that were analyzed in more depth. The Environmental Sciences area accounted for $81 \%$ of the published articles. The journal with more articles published was the Ecological Indicators, although the Journal of Cleaner Production presented better bibliometric indexes. China and the United States of America are the countries with the largest number of publications and, in the Brazilian case; the State University of Campinas is the institution with the largest number of affiliations. It is concluded that the theme is relatively recent, since the publications started in 2003, with a significant concentration between the years 2011 and 2015, when the number of publications quadrupled. The theme addressed in this work presented a high index of replicability in different spheres of sustainability analysis.
\end{abstract}

Keywords: Bibliometrics; Scopus Base; Sustainability; Environmental Management. 


\section{Introdução}

A constante busca do homem por conhecimento traz a necessidade da realização do inventário das atividades científicas nas diversas áreas do conhecimento, disseminando globalmente as informações contidas no universo acadêmico científico. Dentro do universo da gestão da informação e do conhecimento científico e tecnológico, a bibliometria se apresenta como uma ferramenta que por meio de métodos estatísticos é capaz de medir e analisar a produção científica em suas diferentes áreas (SILVA et al., 2009).

Em virtude das crescentes preocupações com o ambiente e da necessidade do estabelecimento de políticas públicas para se atingir o desenvolvimento sustentável, as discussões no campo da Gestão Ambiental se tornam cada vez mais comuns na comunidade científica, destacando-se dentre as diversas áreas do conhecimento ao apresentar um expressivo aumento do número de publicações (ZHONG et al., 2016)

Ao longo das últimas duas décadas, uma lista em contínuo crescimento de indicadores de sustentabilidade foi introduzida na comunidade científica com o objetivo de sensibilizar a opinião pública de como a humanidade exerce pressões sobre o ambiente (FANG et al., 2014). Os Indicadores de sustentabilidade vêm sendo empregados no monitoramento ambiental transmitindo informações sobre o desenvolvimento de uma região ou processo relacionado ao progresso social, econômico e ambiental, como abordado no desenvolvimento sustentável. Nesse contexto, os indicadores Pegada Ecológica (PE) (WACKERNAGEL e REES, 1996) e a Emergia ou Análise Emergética (AE) (ODUM, 1996) se apresentam como conceitos essenciais na análise do nível de sustentabilidade de um determinado local (AGOSTINHO e PEREIRA, 2013)

A PE consiste em uma ferramenta da sustentabilidade que totaliza os fluxos de matéria e energia de entrada e saída de um sistema econômico, convertendo-os em área correspondente de terra ou água existentes na natureza para sustentar o padrão de consumo do sistema analisado (ALVES, 2014). O método da PE questiona a possibilidade de se desenvolver a sociedade sem aumentar a pressão humana sobre o planeta; sem comprometer as gerações futuras, a partir da mensuração do impacto de uma população sobre o ambiente. Apesar de ser uma ferramenta criada na década de 90, a PE se popularizou durante os últimos anos, quando houve crescimento expressivo de suas aplicações e influência na sociedade, principalmente na implementação de políticas públicas, planejamento, e monitoramento das agendas de desenvolvimento sustentável de diversas nações (SEVERO e DE GUIMARÃES, 2017)

A PE tem sido usada para definir metas e direcionar mudanças no orçamento, investimentos sustentáveis e política em diversas cidades e em países como a Alemanha, Bélgica, Canadá, Inglaterra, Japão, País de Gales, e Suíça. Além disso, empresas privadas como BASF (Brasil), BC Hydro (Canadá), SITA (França), entre outras, vem utilizando essa ferramenta na busca pela redução de seus impactos ambientais (WHITBY, 2014).

Por outro lado, o conceito de emergia pode ser entendido como uma ferramenta que contempla uma análise simultânea dos sistemas ecológicos e econômicos permitindo identificar e quantificar o uso de recursos naturais, compreender os limites de cada ecossistema analisado, possibilitando a definição de metas para garantir a capacidade de suporte e, portanto, sua sustentabilidade (ODUM, 1996).

A combinação entre os dois métodos tem como produto a Pegada Ecológica Emergética (PEE) que pode ser usada para amparar decisões por parte dos governos, instituições públicas e privadas, visando atingir um padrão de desenvolvimento sustentável na sociedade. A PEE é empregada para estimar a valoração do trabalho realizado pela natureza, a capacidade de suporte dos ecossistemas e contabilização dos impactos ambientais causados por um recurso, processo ou serviço. Contemplando assim uma análise simultânea dos sistemas ecológicos, econômicos e 
sociais (CHEN e CHEN, 2006; NAKAJIMA e ORTEGA, 2016; PEREIRA e ORTEGA, 2012; SICHE et al., 2008; ZHAO et al., 2005)

Além de serem consideradas importantes ferramentas didáticas na área de Educação Ambiental, são também indicadores do impacto ambiental causado pelas atividades antrópicas sobre o meio ambiente, servindo como base para tomada de decisões por parte de instituições públicas e privadas na gestão dos recursos naturais (PEREIRA e ORTEGA, 2012)

Visando contribuir com o conhecimento na área de Sustentabilidade e Gestão Ambiental, permitindo ao leitor a compreensão de sua complexidade e importância para a comunidade científica, o objetivo deste trabalho consiste em disseminar o conhecimento acerca do tema PE sob a ótica da emergia. A partir de uma revisão bibliométrica sistematizada foram apresentados indicadores bibliométricos que permitam mapear seu estado da arte na base SCOPUS.

\section{Revisão da Literatura}

\subsection{Bibliometria}

O estudo bibliométrico consiste em um método efetivo para analisar quantitativamente publicações acadêmicas utilizando técnicas estatísticas (VANTI, 2002). A revisão bibliométrica pode ser entendida como o estudo de técnicas e métodos voltados para o desenvolvimento de indicadores de publicações do universo cientifica que busca relacionar o levantamento bibliográfico a estatística (COSTA, 2016) Esta ferramenta é empregada na análise sistemática do conhecimento em diversas áreas da ciência, com o objetivo de avaliar o estado da arte e tendências de um determinado assunto, assim como identificar direções para estudos futuros, servindo de guia para novos pesquisadores (ZHANG et al., 2010; ZHONG et al., 2016).

Existem três autores que se destacaram por suas importantes contribuições na área de bibliometria: Lotka, Zipf e Bradford. Cada um destes pesquisadores pode ser identificado com uma "lei" específica (Quadro 1). A Lei de Lotka, popularmente conhecida como "Lei do Quadrado Inverso", demonstra a medição da produtividade dos autores, mediante um modelo de distribuição tamanho-freqüência dos diversos autores em um conjunto de documentos publicados (DE CARVALHO PEREIRA et al., 2015). A Lei de Zipf, também conhecida como "Lei do Mínimo Esforço", descreve a relação entre palavras num determinado texto e a ordem de série destas palavras, gerando uma lista em ordem decrescente de frequência de aparecimento destas. A posição de uma palavra nesta lista é multiplicada pela sua frequência, igualando uma constante. Já a Lei de Bradford, ou Lei de Dispersão, estuda os conjuntos de periódicos mediante a mensuração da produtividade destas, estabelecendo o núcleo e as áreas de dispersão sobre um determinado assunto em um mesmo conjunto (VANTI, 2002).

\begin{tabular}{|l|l|l|}
\hline $\begin{array}{l}\text { Leis Clássicas da } \\
\text { Bibliometria }\end{array}$ & Foco de Estudo & Principais aplicações \\
\hline Lei de Lotka & Autores & $\begin{array}{l}\text { Estimar o grau de relevância de autores, em dada área } \\
\text { do conhecimento. }\end{array}$ \\
\hline Lei de Zipf & Palavras & $\begin{array}{l}\text { Indexação automática de artigos científicos e } \\
\text { tecnológicos. }\end{array}$ \\
\hline Lei de Bradford & $\begin{array}{l}\text { Estimar o grau de relevância de periódicos, em dada } \\
\text { área do conhecimento. }\end{array}$ \\
\hline $\begin{array}{l}\text { Quadro 1: As Leis Clássicas da Bibliometria de acordo com o foco de estudo e aplicações. Fonte: } \\
\text { Elaborado pelos autores (2018). }\end{array}$ \\
\hline
\end{tabular}

\subsection{Aplicações bibliométricas}


Essa ferramenta pode ser utilizada para diversos fins, sendo capaz de gerar parâmetros de produção científica de um determinado país. A Bibliometria, como área de estudo da ciência da informação, exerce um papel fundamental na análise da produção científica de um país, uma vez que seus indicadores retratam o grau de desenvolvimento de uma área do conhecimento (JÚNIOR, 2013).

Vanti (2002) define algumas das possibilidades de aplicações das técnicas bibliométricas nas quais se destacam: Identificação das tendências e crescimento do conhecimento em uma área; Identificação dos principais periódicos de uma área; Avaliação dos processos de citação e cocitação; Previsão de tendências de publicação; Análise da produtividade de autores, organizações e países; Medição do grau de colaboração entre autores; Avaliação dos aspectos estatísticos da linguagem, das palavras e frases; Identificação da dispersão e a obsolescência da literatura científica.

Strehl (2005) ressalta a importância da existência ainda de um indicador bibliométrico desenvolvido a partir de dados de citações do Journal Citation Reports (JCR). O JCR é uma base que concentra os periódicos mais relevantes das diversas áreas científicas desde 2001. A seleção dos periódicos nesta base é realizada pela avaliação do Fator de Impacto, em uma base de revistas de 3.300 editores, cerca de 200 disciplinas, e 60 países. Anualmente, são publicados no JCR três indicadores por título de periódico: O índice de citação imediata (immediacy index), a meia-vida das citações (cited Half Life) e o índice do fator de impacto (impact Factor).

\subsection{Base Scopus}

A pesquisa científica é uma tarefa complexa e trabalhosa. Encontrar o resultado correto é tão fundamental quanto descobrir fontes e colaboradores, tendências e análise de resultados. À medida que a pesquisa se torna cada vez mais globalizada, interdisciplinar e colaborativa, é necessário garantir que a as publicações a cerca de um determinado assunto que está sendo estudado não passe despercebido.

A Scopus é considerada maior base de dados multidisciplinar de resumos e citações de literatura revisada por pares. Criada pela editora Elsevier em 2004, apresenta uma coletânea de publicações desde 1960 e é atualizada diariamente. Atualmente, contém mais de 54 milhões de registros, 22.000 títulos e 5.000 editores. Fornece ainda o panorama mais completo da produção científica mundial nas áreas de ciência, tecnologia, medicina, ciências sociais e Artes e Humanidades. Esta base conta com ferramentas inteligentes para rastrear, analisar e visualizar pesquisas com uma importante fonte para comunidade acadêmica que almeja buscar e avaliar a literatura científica. A tabela apresenta algumas das funcionalidades apresentadas pela sua interface

\begin{tabular}{|c|}
\hline Funcionalidades da interface da base SCOPUS \\
\hline Análise da produção científica de autores \\
\hline Análise e Refinamento dos resultados \\
\hline Avaliação tendências nos resultados \\
\hline Exportação dados para Gerenciadores de Referências \\
\hline Link para artigos completos \\
\hline Pesquisa por documento, autor ou afilição \\
\hline Recuperar resultados em massa no formato pdf \\
\hline $\begin{array}{l}\text { Rastreamento de citações ao longo do tempo para um grupo de autores ou } \\
\text { documentos }\end{array}$ \\
\hline Visualização de indicadores bibliométricos de autores e periódicos \\
\hline
\end{tabular}


Esta base conta com ferramentas que ajudam os pesquisadores a fazer descobertas e análises, indo além da pesquisa. A busca básica pode ser feita por assuntos, com delimitação de campos de dados, período de publicação, e áreas do conhecimento. A pesquisa avançada permite a utilização de estratégias complexas, utilizando operadores booleanos e códigos dos campos de dados (SILVA et al., 2009).

\section{Metodologia}

Neste trabalho foi realizado um estudo bibliométrico sobre o tema "Pegada Ecológica e Emergia" em artigos científicos publicados em periódicos indexados na base de dados do Scopus, que se encontra disponível no Portal de Periódicos da Capes. O motivo de escolha desta base foi devido ao potencial de abrangência de publicações que a mesma possui no âmbito acadêmico científico.

A pesquisa foi realizada em Abril de 2018 e foram selecionados documentos publicados até o presente período, sem restrição de datas anteriores. Os refinamentos foram realizados usandose os termos: Ecological Footprint, Emergy e Emergy Analysis. Através da ferramenta "busca rápida" da base Scopus, obteve-se o retorno das publicações que apresentavam os termos presente no título, no resumo ou nas palavras-chaves. O cruzamento dos termos usados foi realizado com o emprego do and (\&) e or com propósito de obter a convergência entre estas áreas do conhecimento, buscando uma análise mais aprofundada dos resultados obtidos. Para verificar as palavras-chaves mais comuns nos documentos avaliados utilizou-se a ferramenta wordle que apresenta de forma ilustrativa os termos mais comuns.

A partir dessa análise, foram abordados os aspectos relevantes da pesquisa, além de uma breve discussão sobre resultados obtidos. A metodologia desta bibliometria foi elaborada baseada nos trabalhos de revisão bibliográfica de COSTA, (2010); DE CARVALHO PEREIRA et al., (2015); ZHONG et al., (2016). Os parâmetros estudados foram: a) refinamento da pesquisa com os termos apontados anteriormente; b) analise do tipo de documento e área de publicação; c) pesquisa de palavras-chaves mais comuns; d) identificação dos periódicos com mais artigos publicados; e) identificação dos principais autores; f) nacionalidade e instituição de vínculo dos autores; g) Identificação dos artigos mais citados e h) avaliar a contribuição de autores brasileiros.

\section{Resultados}

Ao inserir a palavra-chave Ecological Footprint, na coleta de informações por meio da opção de busca rápida foram encontrados 3923 documentos, sendo 2784 artigos científicos publicados nos mais diversos periódicos. Utilizando-se do segundo termo Emergy or Emergetic Analysis encontrou-se 1459 publicações, sendo 1116 artigos em periódicos.

Realizando-se o refinamento da pesquisa, foi inserida a seguinte estrutura no campo de busca de pesquisa avançada: "TITLE-ABS-KEY ("ecological footprint") AND TITLE-ABSKEY("emergy") OR TITLE-ABS-KEY("emergetic")" resultando em 138 publicações, sendo 87 destes, artigos científicos publicados em periódicos.

\subsection{Tipologia e número de documentos}

Após realização da pesquisa, os resultados foram segmentados quanto ao tipo de documento. As publicações mais comuns se enquadram nas categorias: artigos em periódicos e trabalhos de conferências (Tabela 1). 
Tabela 1: Classificação dos tipos de publicações na base Scopus em 2017

\begin{tabular}{lc}
\hline Tipo de Documento & Resultado \\
\hline Artigo Científico & 87 \\
Pôster de Congresso & 32 \\
Revisão & 11 \\
Capítulo de Livro & 6 \\
Amostra Curta & 1 \\
Revisão de Conferência & 1 \\
\hline Fonte: Elaborado pelos autores (2018)
\end{tabular}

Como os artigos representam a maior parte das publicações, estes foram selecionados para um estudo mais aprofundado, incluindo o título do periódico, país da publicação, palavras-chave, autor, ano de publicação e número de citações. Dentre os 87 artigos encontrados, percebe-se que as publicações se iniciaram em 2003, demonstrando que esta temática é relativamente recente para a comunidade científica.

A concentração de artigos ocorreu em dois ciclos de ocorrência, o primeiro entre 2008 e 2010 e o último entre 2014 e 2016 (Figura 1). Percebe-se ainda que o número de publicações aumentou significativamente entre os anos de 2005 ( 1 publicação) e 2008 (10 publicações) e que praticamente quadruplicou entre os anos de 2011 e 2015. Em 2017, houve uma redução de aproximadamente $46 \%$ em relação a 2015 , mas ainda assim é considerado um tema bastante discutido, tanto que para o ano de 2018, ano de realização deste estudo, já haviam três artigos publicados no mês de abril.

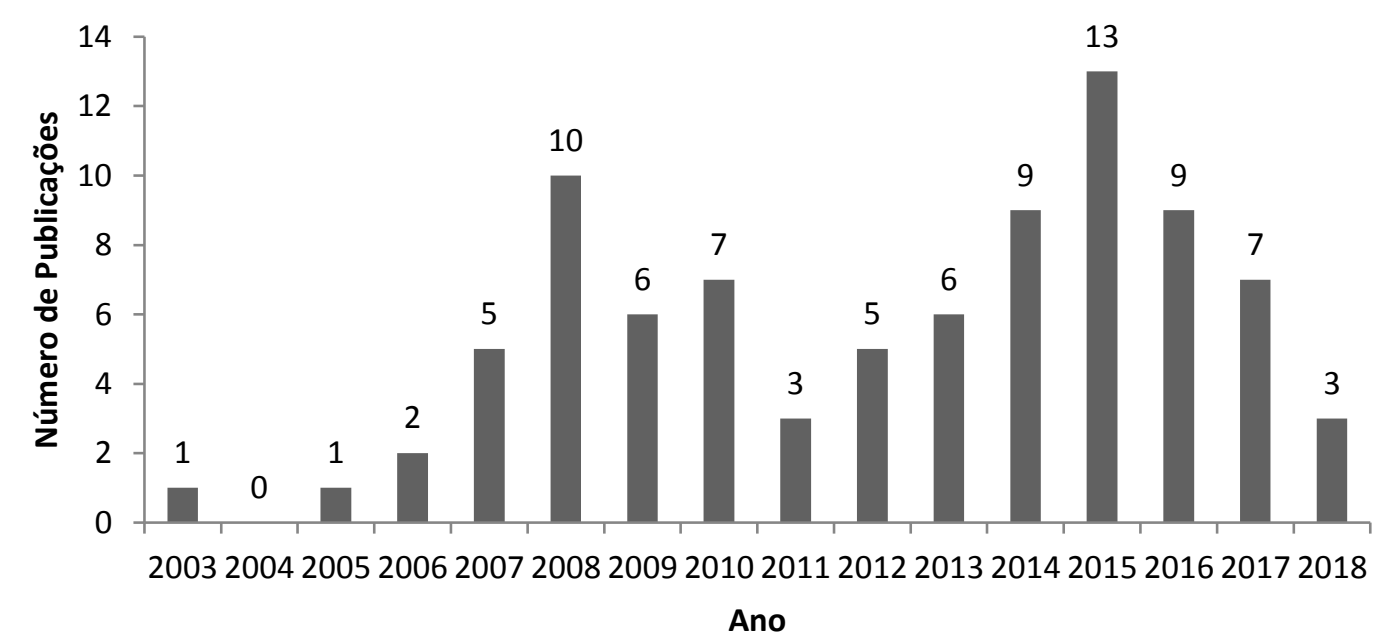

Figura 1: Número de publicações de artigos sobre Pegada Ecológica na base SCOPUS. Fonte: Elaborado pelos autores (2018).

\section{2 Área de concentração}

Neste tópico foram listadas as 10 áreas de interesse com maior número de publicações voltado para esta temática (Tabela 3). Verificou-se que a área de Ciência Ambiental e Agricultura e Ciências Biológicas concentram a maior parte dos artigos publicados. O somatório de registros 


\section{ACTA}

apresentado na Tabela 2 (total de 178 ocorrências) diverge do valor total de artigos publicados (87 ao todo), isso se justifica pelo fato de que alguns artigos são contabilizados em mais de uma área. Observa-se inclusive grande multidisciplinaridade das áreas de interesse voltadas para o assunto em estudo, o que demonstra uma replicabilidade em diferentes esferas de análise.

Tabela 2: Número de publicações por áreas de interesse.

\begin{tabular}{lc}
\hline Área de Interesse & Resultado \\
\hline Ciência Ambiental & 71 \\
Agricultura e Ciências Biológicas & 41 \\
Engenharia & 16 \\
Energia & 13 \\
Negócios, Gestão e Contabilidade & 12 \\
Ciências de decisão & 11 \\
Multidisciplinar & 5 \\
Engenharia Química & 3 \\
Ciência da Computação & 3 \\
Economia, Econometria e Finanças & 3
\end{tabular}

Fonte: Elaborado pelos autores (2018).

\subsection{Identificação das palavras-chaves}

A nuvem de palavras auxilia no reconhecimento das palavras-chaves mais utilizadas em diversos tipos de textos, ela transmite de forma ilustrativa a frequência com que os termos apresentados aparecem entre as palavras chaves dos artigos científicos analisados. Após analisar as palavras-chaves contidas nos artigos, obteve-se 499 termos utilizados para formar a nuvem de palavras (Figura 2).

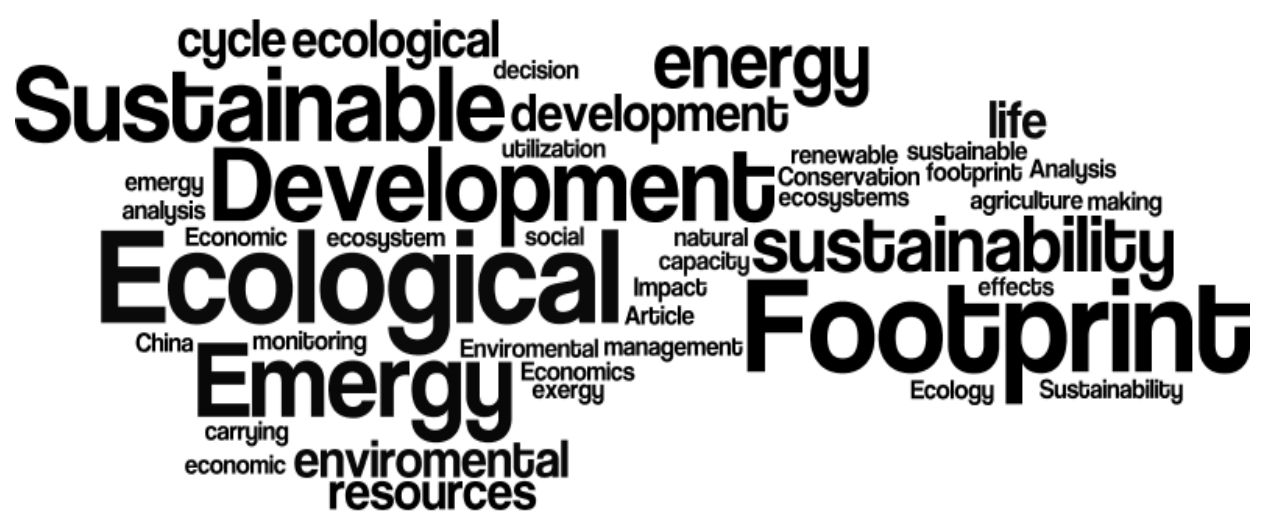

Figura 2: Nuvem de palavras. Fonte: www.wordle.net.

Na Figura 3, verificou-se que os termos "Ecological Footprint" (60 registros), "Emergy" (51 registros), "Sustainable Development" (39 registros), "Sustainability" (38 registros) $e$ "Ecology" (36 registros) foram citados com mais frequência. 


\subsection{Identificação da instituição de filiação e sua nacionalidade}

Para a análise deste item foram listadas as dez instituições com o maior número de artigos encontrados na pesquisa, de acordo com o número de resultados (Figura 3). Verificou-se que a Chinese Academy of Sciences aparece em primeiro lugar com 13 publicações vinculadas a instituição. A Universidade Estadual de Campinas (Unicamp), brasileira, aparece em segundo lugar junto a outras 4 Instituições, seguida de perto por mais uma instituição chinesa, a Shenyang Institute of Applied Ecology com 6 publicações.

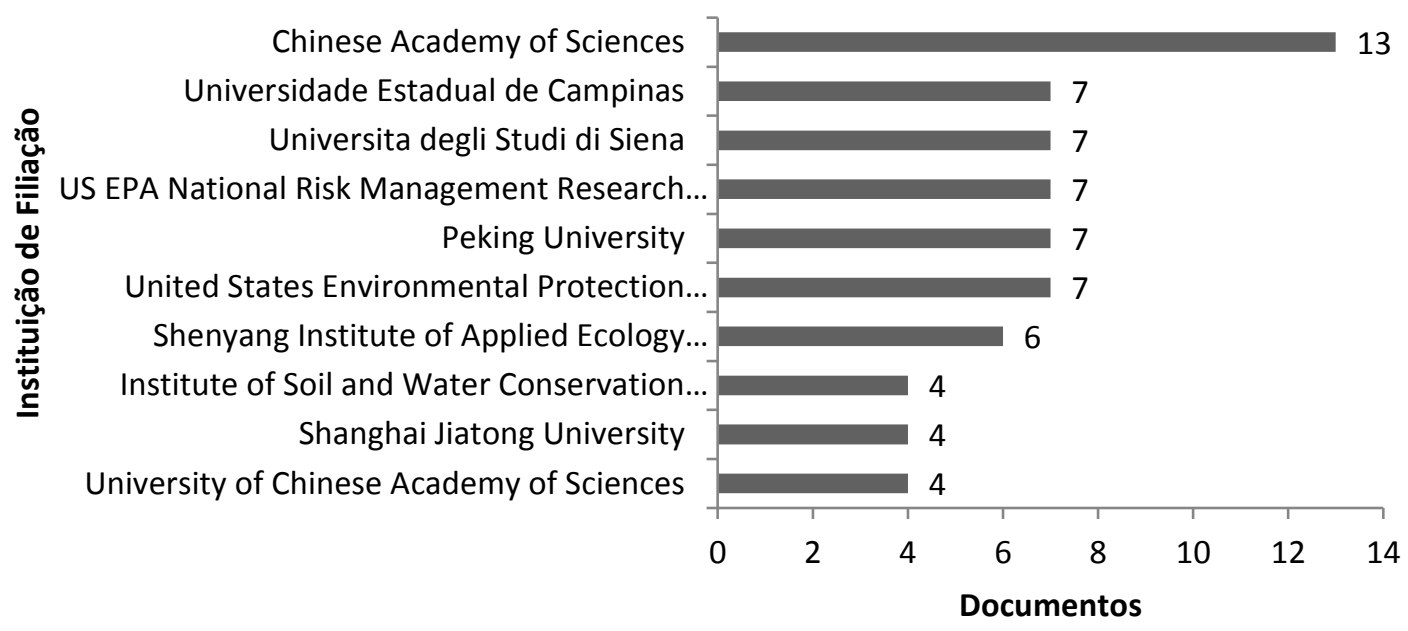

Figura 3: Instituições com maior número de documentos publicados na base Scopus. Fonte: Elaborado pelos autores (2018).

Para uma análise mais detalhada da nacionalidade da instituição de filiação dos autores, foi elaborado de forma gráfica o ranking dos dez países com maior número de vínculos (Figura 4). Percebe-se que a China apresenta a maior concentração de publicações sobre o tema em estudo, atendendo por $57 \%$ do total de artigos analisados. Além disso, é o país que apresentou maior crescimento de pesquisas realizadas acerca do tema de estudo, cerca de $700 \%$ entre os anos de 2005 e 2015.

Em segundo colocado no ranking, aparece os Estados Unidos com 15\% das publicações. Vale destacar ainda presença do Brasil e Itália como os terceiros países com maiores números de publicações. 


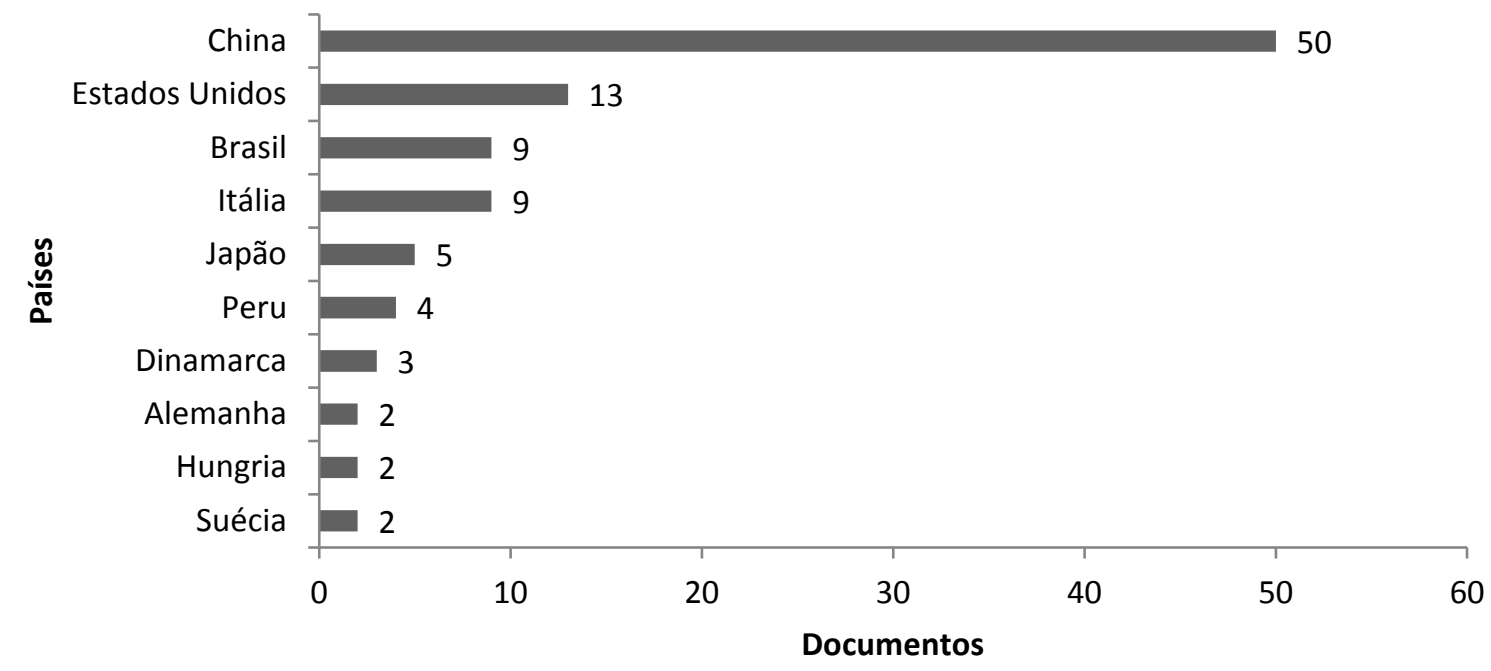

Figura 4: Países com mais filiações de autores. Fonte: Elaborado pelos autores (2018).

\subsection{Identificação dos autores}

Foram classificados os dez autores com o maior número de artigos científicos publicados (Figura 5). Os autores Agostinho, F., Ortega, E., lideram o ranking, com 6 publicações cada. Além disso, esses podem ser considerados os maiores disseminadores do assunto no Brasil, visto que ambos estão vinculados a Universidade Estadual de Campinas.

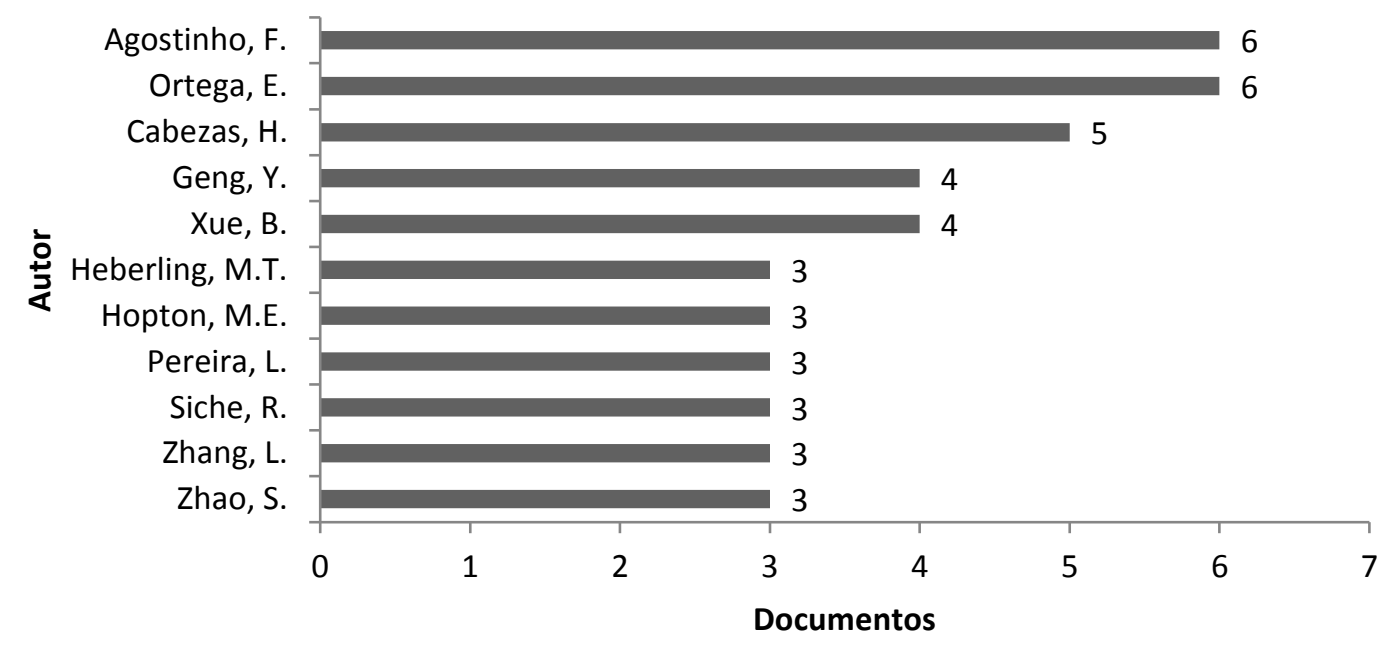

Figura 5: Autores com mais publicações. Fonte: Elaborado pelos autores (2018).

\subsection{Identificação dos principais periódicos}

Para a análise dos periódicos com maior número de artigos publicados na base, foi elaborada uma lista contendo os dez periódicos mais frequentes (Figura 6). Foi possível identificar 
que os periódicos Ecological Indicators, Journal Of Cleaner Production e Shengtai Xuebao Acta Ecologica possuem os maiores números de artigos publicados nesta área.

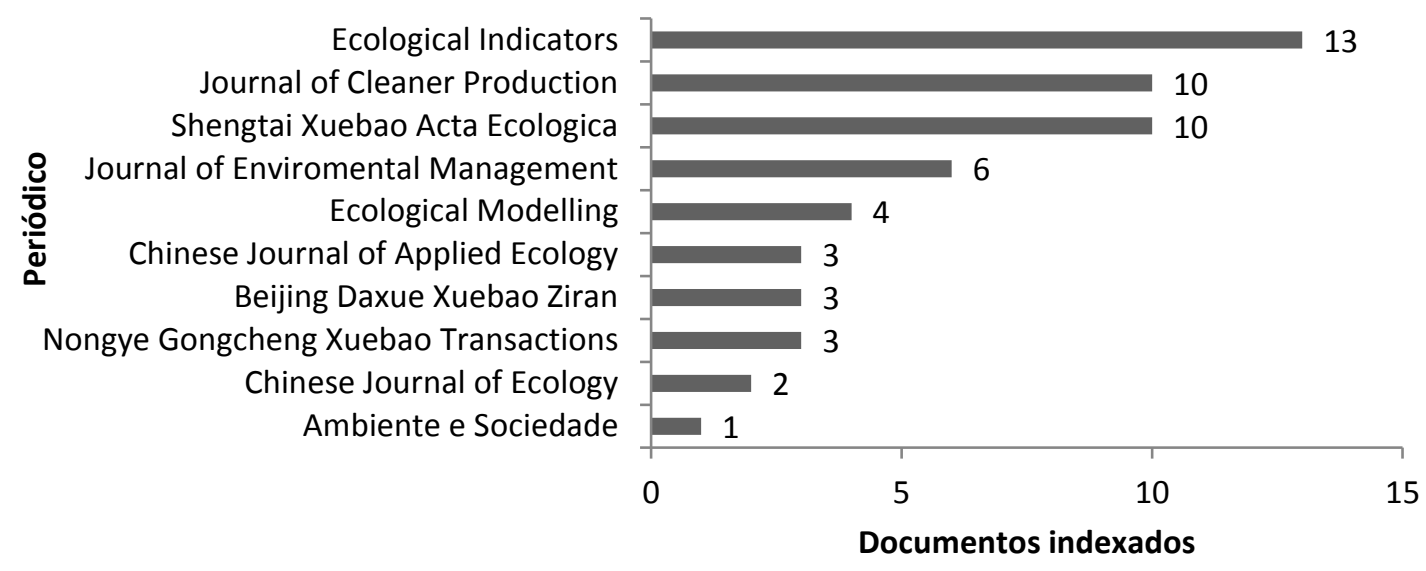

Figura 6: Periódicos com maior número de artigos publicados.

Fonte: Elaborado pelos autores (2018).

Para avaliar a qualidade dos periódicos com o maior número de publicações nas áreas pesquisadas (PE e AE) foi realizada uma análise comparativa de quatro indicadores destes periódicos: Fator de Impacto, número de citações (citescore), SNIP e JCR. O periódico Journal of Cleaner Production apresentou os melhores índices de desempenho em todos os comparativos.

\subsection{Artigos mais citados}

Para identificação dos artigos mais citados na Base Scopus, foram listados os dez artigos mais relevantes sobre o tema abordado (Tabela 3). Verificou-se que os artigos "A modified method of ecological footprint calculation and its application" com 121 citações e "Ecological footprint accounting based on emergy- A case study of the Chinese society" com 104 citações, lideram o ranking dos trabalhos mais citados na comunidade científica, seguido do terceiro colocado "Sustainability of nations by indices: Comparative study between environmental sustainability index, ecological footprint and the emergy performance índices" com 94 citações. Além disso, dentre o conjunto de artigos analisados foi encontrado o $h$-index ou índice-h igual a 16 . Esse indicador representa que foram encontrados pelo menos 16 artigos científicos do total dos documentos analisados foram citados pelo menos 16 vezes. 
Tabela 3: Artigos mais citados na base Scopus referente ao tema Pegada Ecológica Emergética.

\begin{tabular}{l} 
Título do Artigo \\
\hline $\begin{array}{l}\text { A modified method of ecological footprint } \\
\text { calculation and its application }\end{array}$ \\
Ecological footprint accounting based on \\
emergy-A case study of the Chinese society \\
Sustainability of nations by indices: \\
Comparative study between environmental \\
sustainability index, ecological footprint and \\
the emergy performance índices
\end{tabular}

Ecological footprint analysis applied to a subnational area: The case of the Province of Siena (Italy)

The argument against a reductionist approach for measuring sustainable development performance and the need for methodological pluralism

\begin{abstract}
Assessment of economic and ecological carrying capacity of agricultural crops in Nicaragua

Efficiency and sustainability indicators for passenger and commodities transportation systems. The case of Siena, Italy
\end{abstract}

Life cycle assessment of fresh pineapple from Costa Rica

Convergence of ecological footprint and emergy analysis as a sustainability indicator of countries: Peru as case study

20

Ano

2005 Zhao S., Li Z., Ecological

Li W. Modelling

Citações

2006
Chen B., Chen Ecological G.Q. Modelling

Siche J.R.,

Agostinho F., Ecological Ortega E., Economics

Romeiro A.

Bagliani M., Galli A.,

Niccolucci V., Environmental

Marchettini N. Management

2009

$\begin{array}{cc}\text { Gasparatos A., } & \text { Accounting } \\ \text { El-Haram M., } & \text { Forum } \\ \text { Horner M. } & \end{array}$

60

Horner M.

2007

Cuadra M., Ecological

Bjorklund J. Indicators

Federici M.,

Ulgiati S., Ecological

Verdesca D., Indicators

Basosi R.

2012

Ingwersen

Journal of

Cleaner

Production

Communication

Siche R., in Nonlinear

Pereira L., $\quad$ Science and

2010

Agostinho F.,

Numerical

Ortega E. Simulation
Castellini C.,

Boggia A.,

Cortina C.,

A multicriteria approach for measuring the sustainability of different poultry production systems

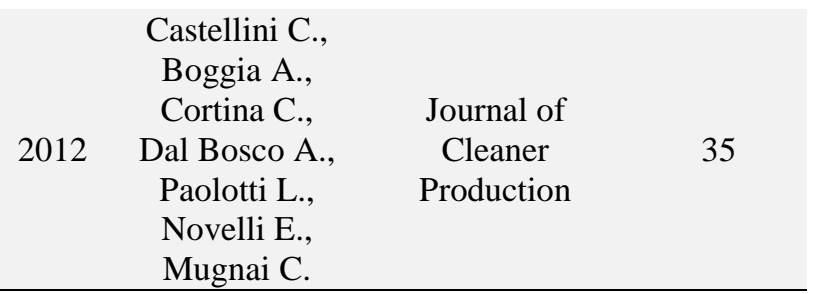

Fonte: Elaborado pelos autores (2018).

Percebe-se que dois artigos, entre os mais citados, foram produzidos por Siche R., Agostinho F., Ortega E. e Pereira, L, que estão vinculados a UNICAMP. Estes autores apresentaram suas contribuições nos periódicos: Ecological Indicators $(\mathrm{n}=4)$, Ambiente e Sociedade, Communications In Nonlinear Science and Numerical Simulation, Ecological Economics, Journal of Cleaner Production, todos respectivamente com uma publicação. 


\section{Discussão}

Com o intuito de realizar uma análise mais detalhada de suas contribuições para a comunidade científica, foram selecionados alguns dos dez artigos mais citados, além daqueles que representaram uma importante contribuição para o tema em estudo.

Zhao et al., (2005) propuseram um método modificado de cálculo da PE baseado nos conceitos da AE. Segundo os autores, o principal objetivo da metodologia proposta é transformar a demanda humana de recursos naturais e a oferta da natureza em conceitos mais compreensíveis e quantificáveis. No cálculo da $\mathrm{BC}$, foi considerado apenas recursos renováveis como fonte de energia. Já no cálculo da PE, os dados de consumo foram transformados em fluxos emergéticos. Por fim o método foi aplicado para a província de Gansu na China, que apresentou resultados mais drásticos em relação ao método convencional da PE.

Chen e Chen (2006) aplicaram a mesma metodologia desenvolvida por Zhao et al. (2005) para avaliar o consumo na sociedade chinesa de 1981 a 2001. Os resultados encontrados na (PEE) deste trabalho representam o nível de consumo de recursos correspondente ao produto econômico unitário. Finalmente, sugere-se que a PEM sirva como um indicador modificado de PE para ilustrar os recursos, o ambiente e a atividade da população, refletindo, assim, o estado da arte do sistema ecológico analisado.

Siche et al. (2008) faz uma comparação entre os dois índices de sustentabilidade aplicados a países: "pegada ecológica" e "índice de sustentabilidade ambiental", a partir do desenvolvimento de dois novos índices emergéticos (renovabilidade e $\mathrm{AE}$ ) que ganharam espaço dentro da comunidade científica e agências governamentais. Concluiu-se que, embora com algumas deficiências, a combinação entre os métodos da PE e emergia, seria a metodologia mais apropriada para explicar a realidade ecológica de um sistema nacional. Os autores ainda sugerem ainda a utilização do Fator Capacidade de Suporte ao invés da determinação do saldo ecológico, o que indicaria se o sistema é sustentável ou não.

Bagliani et al. (2008) visaram investigar a sustentabilidade ambiental da Província de Siena e dos seus municípios, através de diferentes indicadores e métodos de análise. Foram utilizados os métodos da PE e da biocapacidade como indicadores para monitorar as condições ambientais da região. A combinação entre os métodos abordados permitiu investigar em detalhes os aspectos ambientais da região.

Pereira e Ortega (2012) propõe algumas modificações ao método desenvolvido por Zhao et al. (2005) com o objetivo de, não só introduzir mais profundamente o conceito de emergia, mas também incorporar a metodologia da $\mathrm{AE}$ nos cálculos, suprindo as deficiências encontradas. Nesta nova abordagem é adicionada ao cálculo da $\mathrm{BC}$, uma nova categoria de valores "áreas não ocupadas pelo ser humano" as quais costumam ser excluídas nas metodologias anteriores. O método foi aplicado ao Brasil, fazendo um comparativo de valores para PE encontrados com trabalhos anteriores.

Agostinho e Pereira (2013) fazem um estudo comparativo entre os métodos da Energia Incorporada, PE e AE, aplicados ao cálculo da capacidade de suporte. Os autores discutem que o cálculo da área de suporte por meio da AE é mais apropriado para representar o impacto ambiental em escala global. No entanto, cada metodologia apresenta uma faixa diferente de aplicação e que dependendo dos objetivos de estudo, esta terá sua contribuição mais acentuada.

Nakajima e Ortega (2016) avaliaram a capacidade de suporte da região de Ibiúna utilizando a AE para aprimorar o diagnóstico dos problemas ambientais e facilitar a compreensão da sustentabilidade, apoiando a formulação de políticas públicas voltadas para preservação do ambiente.

Na economia clássica, a valoração de um produto se baseia no esforço do trabalho humano agregado e no custo dos insumos necessários para sua produção. Entretanto, nesta não é considerado o trabalho realizado pela natureza e seus ecossistemas, em fornecer as matérias primas 
necessárias para sua produção. Assim como as externalidades negativas ambientais e sociais, como degradação do solo, desmatamento e desequilíbrio ecológico (PEREIRA e ORTEGA, 2012).

A análise emergética realiza uma contabilização de todos os recursos naturais (água, chuva, vento, sol, marés, solo, etc.) e os recursos econômicos (mão de obra, equipamentos industriais, produtos químicos, insumos e máquinas agrícolas) que incidem sobre um ecossistema. Isso só é possível pela transformação do valor econômico em valor emergético solar. A emergia está diretamente associada à fonte primária de energia, o sol, e possui sua unidade de medida padrão denominadas Emergia Solar (seJ) (ODUM, 1996).

As aplicações da AE vão desde áreas de planejamento regional, do uso de terra, a partir de estimativas de extensão de área necessária para cultivo e de preservação ambiental, até a identificação de prioridades de desenvolvimento de agroindústrias que se adequem à realidade sócio-econômica da população, e à capacidade de suporte do meio ecológico (PEREIRA e ORTEGA, 2012)

Apesar de métodos distintos, tanto a PE como a $\mathrm{AE}$ possuem a mesma finalidade: identificar a relação entre a oferta de recursos da natureza e a demanda imposta pelas atividades humanas sobre estes. De acordo com Siche et al. (2008), estes são considerados os indicadores mais apropriados para medir a sustentabilidade de um determinado local ou região, avaliando o fluxo de energia no ambiente e seu estado ambiental. Portanto, a convergência entre os dois métodos suprem possíveis falhas em suas metodologias, e proporcionam, assim, um indicador mais completo e robusto, fornecendo um panorama mais exato do nível de sustentabilidade de determinado local e sua realidade ecológica (WIEDMANN e BARRETT, 2010).

A PEE vem sendo utilizada em escalas cada vez maiores para avaliar as entradas (inputs) e saídas (outputs) de sistemas ecológicos, assim como suas externalidades. A PEE consiste em um método mais completo que a PE sob a ótica ecológica, por considerar fluxos de energias adquiridas na produção de produtos e serviços. A metodologia estima valores das energias naturais incorporadas aos produtos, processos e serviços, as quais, geralmente, não são contabilizadas na economia clássica (SICHE et al., 2008).

\section{Considerações finais}

As publicações nesta área são recentes, tendo início em 2003, até abril de 2018 foram encontrados 87 artigos científicos publicados nos mais diversos periódicos. Entre os anos de 2011 e 2015 houve a maior taxa de crescimento do número de publicações, pois se quadruplicara neste período. As publicações se concentraram nas áreas de Ciências Ambientais, Agricultura e Ciências Biológicas e Engenharia. Os periódicos com maiores números de trabalhos científicos sobre o tema foram: Ecological Indicator, Journal of Cleaner Production e Acta Ecologica Sinica.

A China e os Estados Unidos da América são os países que mais possuem artigos publicados nesta área, tendo a China o grande destaque possuindo 57\% do total das publicações, o que retrata a relevância do conhecimento disseminado desta área para comunidade científica. $\mathrm{O}$ Brasil e a Itália aparecem em terceiro lugar com oito publicações no ranking dos países com maior número de publicações acerca do tema. As contribuições brasileiras acerca do tema se concentram na Unicamp, sendo considerada a instituição com maior número de artigos vinculados juntamente com a Universita degli Studi di Siena. Quanto aos periódicos, as publicações dos autores brasileiros se concentraram nas revistas Ecological Indicators e Ambiente \& Sociedade.

Contudo, foi possível avaliar o estado da arte e tendências do tema PE sob a ótica da Emergia, sendo verificado que o tema exerce papel importante na comunidade científico. Verificou-se ainda que a convergência entre as duas metodologias abordadas neste estudo apresentou alto índice de replicabilidade em diferentes esferas de análises. Identificaram-se também direções para estudos futuros fazendo com que este trabalho sirva de guia para novos pesquisadores da temática abordada. Para futuros trabalhos, sugere-se a utilização de outras bases 
de dados como Web of Science, Scielo, entre outras, o que permitiria aumentar a amostra a ser analisada.

\section{Agradecimentos}

Agradecemos ao Instituto Federal Fluminense pela concessão da bolsa de estudos, auxiliando no desenvolvimento do estudo.

\section{Referências}

AGOSTINHO, F.; PEREIRA, L. Support area as an indicator of environmental load: Comparison between Embodied Energy, Ecological Footprint, and Emergy Accounting methods. Ecological Indicators, v. 24, p. 494-503, jan. 2013.

ALVES, J. E. D. Sustentabilidade, Aquecimento Global e o Decrescimento Demo-Econômico / Sustainability, Global Warming and the Demo-Economic Degrowth. Revista Espinhaço (UFVJM), v. 3, n. 1, p. 4-16, 1 jun. 2014.

BAGLIANI, M.; GALLI, A.; NICCOLUCCI,V.; MARCHETTINI, N. Ecological footprint analysis applied to a sub-national area: The case of the Province of Siena (Italy). Journal of Environmental Management, v. 86, n. 2, p. 354-364, jan. 2008.

CHEN, B.; CHEN, G. Q. Ecological footprint accounting based on emergy-A case study of the Chinese society. Ecological Modelling, v. 198, n. 1-2, p. 101-114, set. 2006.

COSTA, H. G. Modelo para webibliomining: proposta e caso de aplicação. Revista da FAE, v. 13, n. 1, p. 115-126, 2010.

DE CARVALHO PEREIRA, F.; VEROCAI, H. D.; CORDEIRO, V. R.; GOMES, C. F.; COSTA, H. G Bibliometric analysis of Information Systems related to Innovation. Procedia Computer Science, v. 55, p. 298-307, 2015.

FANG, K.; HEIJUNGS, R.; DE SNOO, G. R. Theoretical exploration for the combination of the ecological, energy, carbon, and water footprints: Overview of a footprint family. Ecological Indicators, v. 36, p. 508-518, jan. 2014.

JÚNIOR, C. M; SOUZA, M. T. S.; RIBEIRO, H. C. M.; FURLANETO, C. J.; SILVA, W. N, CAMPANARO, P. M. Estudo bibliométrico da sustentabilidade ambiental: os 15 anos do Simpósio de Administração da Produção, Logística e Operações Internacionais - SIMPOI, Journal of the Health Sciences Institute, São Paulo, 2013.

NAKAJIMA, E. S.; ORTEGA, E. Carrying capacity using emergy and a new calculation of the ecological footprint. Ecological Indicators, v. 60, p. 1200-1207, jan. 2016.

ODUM, H. T. Environmental accounting: EMERGY and environmental decision making. New York: Wiley, 1996.

PEREIRA, L.; ORTEGA, E. A modified footprint method: The case study of Brazil. Ecological Indicators, v. 16, p. 113-127, maio 2012 
SEVERO, E. A.; DE GUIMARÃES, J. C. F. Trajetórias e perspectivas da sustentabilidade e práticas ambientais: uma pesquisa bibliométrica. Revista Metropolitana de Sustentabilidade (ISSN 2318-3233), v. 7, n. 2, p. 93-114, 2017.

SICHE, J. R.; F. AGOSTINHO, ORTEGA, E. \& ROMEIRO, A. Sustainability of nations by indices: Comparative study between environmental sustainability index, ecological footprint and the emergy performance indices. Ecological Economics, v. 66, n. 4, p. 628-637, jul. 2008.

SILVA, A. J.; TOLEDO FILHO, J. R.; PINTO, J. Análise bibliométrica dos artigos sobre controladoria publicados em periódicos dos programas de pós-graduação em ciências contábeis recomendados pela capes. Associação Brasileira de Custos - ABCustos, v. 4, n. 1, p. 36-52, jan./abr. 2009.

STREHL, L. O fator de impacto do ISI e a avaliação da produção científica: aspectos conceituais e metodológicos. Ciência da Informação, Brasília, v. 34, n. 1, p. 19-27, jan./abr. 2005.

VANTI, N. A. P. Da bibliometria à webometria: uma exploração conceitual dos mecanismos utilizados para medir o registro da informação e a difusão do conhecimento. Ciência da Informação, Brasília, v. 31, n. 2, p. 152-162, maio/ago. 2002.

WACKERNAGEL, M.; REES, W. Our Ecological Footprint: Reducing Human Impact on the Earth. New Society Publishers, 1996.

WHITBY, A. The BRAINPOoL Project: Beyond GDP - From Measurement to Politics and Policy. BRAINPOoL deliverable 5.2, mar. 2014.

WIEDMANN, T.; BARRETT, J. A Review of the Ecological Footprint Indicator-Perceptions and Methods. Sustainability, v. 2, n. 6, p. 1645-1693, 7 jun. 2010.

ZHANG, Y.; SINGH, S.; BAKSHI, B. R. Accounting for Ecosystem Services in Life Cycle Assessment, Part I: A Critical Review. Environmental Science \& Technology, v. 44, n. 7, p. 2232-2242, abr. 2010.

ZHAO, S.; LI, Z.; LI, W. A modified method of ecological footprint calculation and its application. Ecological Modelling, v. 185, n. 1, p. 65-75, jun. 2005.

ZHONG, S. A; GENG, Y.; LIU, W.; GAO, C.; CHEN, W. bibliometric review on natural resource accounting during 1995-2014. Journal of Cleaner Production, v. 139, p. 122-132, dez. 2016. 